\title{
Valor Nutritivo de Silagens de Híbridos de Sorgo (Sorghum bicolor (L.) Moench) sob Doses Crescentes de Adubação ${ }^{1}$
}

\section{Magno José Duarte Cândido², José Antônio Obeid ${ }^{3}$, Odilon Gomes Pereira ${ }^{3}$, Paulo Roberto Cecon ${ }^{4}$, Augusto César de Queiroz ${ }^{3}$, Mário Fonseca Paulino ${ }^{3}$, Miguel Marques Gontijo Neto ${ }^{2}$}

RESUMO - Avaliou-se o valor nutritivo das silagens de cinco híbridos de sorgo (AG2002, AG2005E, AGX202, AGX213 e AGX215), cultivados sob doses crescentes de adubação (tratamento sem adubação, 0,5; 1,0; e 2,0 vezes a recomendação de adubação, consistindo de 0; 45,5; 91; e $182 \mathrm{~kg} /$ ha de N; 0; 49; 98; e $196 \mathrm{~kg} /$ ha de $\mathrm{P}_{2} \mathrm{O}_{5}$; e 0; 14; 28; e 56 kg/ha de $\mathrm{K}_{2} \mathrm{O}$, além de 0; 42; 84; e 168 kg/ha de S, respectivamente, advindos da utilização de sulfato de amônio em cobertura), adotando-se o esquema fatorial $5 \times 4$ no delineamento em blocos casualizados, com quatro repetições. O plantio foi realizado no dia 06.11.1997, e as parcelas foram colhidas com os grãos no estádio farináceo. De cada parcela útil retirou-se uma amostra para enchimento dos silos experimentais, que, após 60 dias, foram abertos e amostrados novamente para se proceder à pré-secagem e às determinações do valor nutritivo (PB, fração fibrosa e DIVMS). Os dados foram interpretados por análise de variância e regressão, desdobrando-se a interação híbrido $\mathrm{x}$ adubo independentemente da sua significância. Os fatores qualitativos foram comparados, utilizando-se o teste de Tukey. Os modelos foram escolhidos, baseando-se na significância dos coeficientes de regressão, utilizando o teste de "t", Student, a 10\% de probabilidade, e no coeficiente de determinação. $\mathrm{O}$ valor nutritivo das silagens diferiu entre os híbridos, observando-se maior valor nutritivo para a silagem do AG2005E, enquanto as silagens do AG2002 e do AGX213 apresentaram maior produção de nutrientes por unidade de área. O valor nutritivo das silagens foi afetado pelas doses de recomendação de adubação, embora de forma inconsistente.

Palavras-chave: adubação, silagem, sorgo, valor nutritivo

\section{Nutritive Value of Five Sorghum Hybrids (Sorghum bicolor (L.) Moench) Silages under Increasing Fertilization Levels}

\begin{abstract}
The nutritive value of five sorghum hybrids (AG-2002, AG-2005E, AG-X202, AG-X213 and AG-X215) silages, cultivated under increasing fertilization levels (control, 0.5, 1.0, and 2.0 times of the recommended fertilization, corresponding to $0,45.5$, 91, and $182 \mathrm{~kg} / \mathrm{ha} \mathrm{N} ; 0,49,98$, and $196 \mathrm{~kg} / \mathrm{hade} \mathrm{P}_{2} \mathrm{O}_{5}$; and 0, 14, 28, and $56 \mathrm{~kg} /$ ha de $\mathrm{K}_{2} \mathrm{O}$, besides of 0, 42, 84, and 168 kg/ha S, respectively, that came from the used ammonia sulfate). A complete randomized block design in a factorial $5 \times 4$ arrangement (five hybrids and four fertilization levels) with four replicates was used. The seeding time was at 11.06.1997 and plots were harvested at the soft dough stage of grain. From each plot, a sample was collected to fill up the experimental silos, which were opened after 60 days and sampled again to determine the pre drying weight and the nutritive value (CP, fiber fractions and IVDMD). Data were interpreted by means of analysis of variance and regression. The hybrid $\mathrm{x}$ fertilizer interaction was unrolled independently of its significance. The qualitative factor means were compared by Tukey test. The models were chosen based on regression coefficients significance, using Student " $t$ " test, at $10 \%$ level, and on the determination coefficient. The silages nutritive value differed among the hybrids. AG2005E showed the highest nutritive value, whereas AG2002 and AGX213 showed the highest nutrient yield per unit of area. The fertilizers affected the silages nutritive value, but, inconsistently.
\end{abstract}

Key Words: fertilization, nutritive value, silage, sorghum

\section{Introdução}

O uso de forragens conservadas na forma de silagem está se tornando cada vez mais comum, principalmente entre os produtores de leite, como uma alternativa para amenizar o problema de escassez de pasto no período de estiagem (Pereira et al., 1993). Com a intensificação dos sistemas de produção, cresceu a demanda por silagens que associem elevados rendimento forrageiro e valor nutritivo, maximizando a produção de nutrientes por unidade de área.

Como forrageiras que melhor se adequam à prática da ensilagem encontram-se o milho (Zea mays L.) e o sorgo (Sorghum bicolor (L.) Moench). Apesar de a silagem de sorgo ser considerada de valor nutritivo inferior à de milho, sua ensilagem vem

\footnotetext{
1 Parte da tese de mestrado do primeiro autor.

2 Doutorando em Zootecnia/UFV. Av. P. H. Rolfs, s/n, 36.571-000, Viçosa-MG. E.mail: magno@alunos.ufv.br; mgontijo@cnpgc.embrapa.br

3 Prof. Dep. de Zootecnia/UFV. Av. P. H. Rolfs, s/n, 36.571-000, Viçosa-MG. E.mail: jaobeid@ufv.br

${ }^{4}$ Prof. Dep. de Informática/UFV.
} 
ganhando destaque, pois essa cultura apresenta maior tolerância à seca que o milho, devido ao seu sistema radicular mais abundante e profundo; apresenta rebrotas após os cortes, obtendo-se até $60 \%$ da produção do primeiro corte; não concorre com a alimentação humana, sendo, portanto, de custo mais reduzido; e algumas variedades e, ou, híbridos de sorgo apresentam maior produção de matéria seca e matéria verde por unidade de área que o milho (Resende, 1991; Tonani, 1995; Zago, 1999).

A grande demanda por materiais de melhor qualidade favoreceu o surgimento de inúmeros genótipos, com características específicas de porte (alto, médio, baixo), ciclo (precoce ou tardio) e aptidão (forrageiro, duplo-propósito, granífero), as quais têm influência marcante no valor nutritivo da silagem produzida. Os novos exemplares de sorgo lançados no mercado devem ser sempre avaliados entre si, independentemente de sua aptidão, para se obterem resultados mais consistentes em relação ao seu potencial para produção de silagem de alto valor nutritivo.

Apesar do grande potencial produtivo da cultura, observam-se produções irregulares nas diversas regiões, em decorrência da não-utilização dos cultivares/híbridos mais adaptados às condições edafoclimáticas locais e também da não correção de deficiências nutricionais no solo, o que é crítico para uma cultura que será colhida integralmente, exportando grande quantidade de nutrientes.

Assim, pode-se inferir que a maior produção de matéria seca em decorrência de uma adubação equilibrada e de um manejo adequado do solo e da cultura, associada a sorgos do tipo granífero ou de duplopropósito, poderia proporcionar maior produção de grãos e maior participação desses na matéria seca total da forragem, elevando, em tese, o valor nutritivo da silagem.

O presente estudo foi realizado com o objetivo de avaliar o valor nutritivo das silagens de cinco híbridos de sorgo, de diferentes aptidões, portes e suculências de colmo, cultivados sob quatro doses de adubação.

\section{Material e Métodos}

Um trabalho preliminar de campo envolvendo a avaliação de cinco híbridos de sorgo para produção de silagem foi realizado no período de 06.11.1997 a 18.02.1998 (Gontijo Neto, 1999), nas dependências da Central de Experimentação, Pesquisa e Extensão do Triângulo Mineiro (CEPET/UFV), no município de Capinópolis-MG, com latitude Sul de 18 $41^{\prime}$ e longitude Oeste de $49^{\circ} 34^{\prime}$ e altitude média de 620,20 m. O clima é o Aw, segundo a classificação proposta por Köppen, quente e úmido, com temperatura do mês mais frio acima de $18^{\circ} \mathrm{C}(\mathrm{A})$, com estação chuvosa no verão e seca no inverno $(\mathrm{w})$. Apresenta precipitações médias anuais entre 1.400 e $1.600 \mathrm{~mm}$. Dados relativos à temperatura e pluviosidade no período experimental encontram-se dispostos na Tabela 1.

A área utilizada apresenta relevo suave ondulado e solo classificado como Latossolo Roxo eutrófico, textura argilosa, desenvolvido a partir do basalto. Nos anos anteriores, a referida área foi utilizada em ensaios com soja.

Em função da análise do solo (Tabela 2) e com base na recomendação obtida pelo método do $\mathrm{Al}^{3+}$,

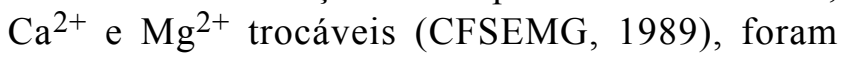
incorporados $400 \mathrm{~kg} /$ ha de calcário $(\mathrm{PRNT}=75 \%)$ ao solo.

O preparo do solo consistiu de uma aração e duas gradagens para destorroamento e nivelamento, sendo a abertura dos sulcos e a distribuição e incorporação do adubo e das sementes efetuadas manualmente. A adubação recomendada para plantio e cobertura baseou-se nas "Recomendações para o Uso de Corretivos e Fertilizantes em Minas Gerais" (CFSEMG, 1989), com pequena variação em razão da utilização de formulações comerciais existentes, e consistiu de $350 \mathrm{~kg} / \mathrm{ha}$ da fórmula (NPK) 06-28-08 no plantio mais $350 \mathrm{~kg} / \mathrm{ha}$ de sulfato de amônio em cobertura, em duas parcelas iguais.

Os cinco híbridos avaliados foram: 1) AG2002 forrageiro, porte alto, ciclo médio de 105 a 110 dias em regiões tropicais, colmo suculento e população recomendada de $125.000 \mathrm{pl} / \mathrm{ha}$; 2) AG2005E - duplopropósito, porte baixo, ciclo precoce (90 a 105 dias), colmo seco e população recomendada de $150.000 \mathrm{pl} / \mathrm{ha}$; 3) AGX202 - forrageiro, porte alto, ciclo médio, colmo seco e população recomendada de $125.000 \mathrm{pl} / \mathrm{ha}$; 4) AGX213 - forrageiro, porte alto, ciclo médio, colmo seco e população recomendada de $125.000 \mathrm{pl} / \mathrm{ha}$; e 5) AGX215 - forrageiro, porte médio/alto, ciclo médio, colmo seco e população recomendada de $150.000 \mathrm{pl} / \mathrm{ha}$. Os dois primeiros são híbridos comerciais e os outros três estão em fase experimental.

As doses de recomendação de adubação utilizadas no experimento foram: 1 - sem adubação de plantio e cobertura; 2 - metade da adubação recomendada para plantio e cobertura $(45,5 \mathrm{~kg}$ de N, $49 \mathrm{~kg}$ de $\mathrm{P}_{2} \mathrm{O}_{5}$ e $14 \mathrm{~kg}$ de $\mathrm{K}_{2} \mathrm{O} / \mathrm{ha}$, mais $42 \mathrm{~kg}$ de $\mathrm{S} / \mathrm{ha}$ oriundos do sulfato de amônio); 3 - uma vez a aduba- 
Tabela 1 - Precipitação e médias das temperaturas máximas e mínimas durante o período experimental Table 1 - Rainfall and maximum and minimum mean temperatures during the experimental period

\begin{tabular}{|c|c|c|c|c|}
\hline \multirow[t]{2}{*}{$\begin{array}{l}\text { Período } \\
\text { Period }\end{array}$} & \multirow[t]{2}{*}{$\begin{array}{l}\text { Semana } \\
\text { Week }\end{array}$} & \multirow[t]{2}{*}{$\begin{array}{c}\text { Precipitação }(\mathrm{mm})^{1} \\
\text { Rainfall }\end{array}$} & \multicolumn{2}{|c|}{$\begin{array}{c}\text { Temperatura }\left({ }^{\circ} \mathrm{C}\right)^{2} \\
\text { Temperature }\left({ }^{\circ} \mathrm{C}\right)^{2}\end{array}$} \\
\hline & & & $\begin{array}{l}\text { Máxima } \\
\text { Maximum }\end{array}$ & $\begin{array}{l}\text { Mínima } \\
\text { Minimum }\end{array}$ \\
\hline $06.11 .97 \mathrm{a} 12.11 .97$ & $1^{\mathrm{a}}$ & 17,0 & 34,8 & 21,9 \\
\hline 13.11 .97 a 19.11 .97 & $2^{\mathrm{a}}$ & 62,4 & 32,3 & 20,9 \\
\hline 20.11 .97 a 26.11 .97 & $3^{\mathrm{a}}$ & 80,8 & 32,1 & 20,9 \\
\hline $27.11 .97 \mathrm{a} 03.12 .97$ & $4^{\mathrm{a}}$ & 188,6 & 30,0 & 21,0 \\
\hline 04.12 .97 a 10.12 .97 & $5^{\mathrm{a}}$ & 67,2 & 28,9 & 20,2 \\
\hline 11.12 .97 a 17.12 .97 & $6^{\mathrm{a}}$ & 65,3 & 30,5 & 21,0 \\
\hline 18.12 .97 a 24.12 .97 & $7^{\mathrm{a}}$ & 8,7 & 30,5 & 20,9 \\
\hline $25.12 .97 \mathrm{a} 31.12 .97$ & $8^{\mathrm{a}}$ & 60,7 & 33,8 & 21,8 \\
\hline $01.01 .98 \mathrm{a} 07.01 .98$ & $9^{\mathrm{a}}$ & 12,7 & 34,5 & 21,8 \\
\hline 08.01 .98 a 14.01 .98 & $10^{\mathrm{a}}$ & 102,2 & 31,6 & 21,9 \\
\hline $15.01 .98 \mathrm{a} 21.01 .98$ & $11^{\mathrm{a}}$ & 40,8 & 30,8 & 20,8 \\
\hline $22.01 .98 \mathrm{a} 28.01 .98$ & $12^{\mathrm{a}}$ & 22,0 & 30,8 & 20,4 \\
\hline $29.01 .98 \mathrm{a} 04.02 .98$ & $13^{\mathrm{a}}$ & 46,8 & 31,1 & 21,8 \\
\hline 05.02 .98 a 11.02 .98 & $14^{\mathrm{a}}$ & 62,6 & 34,2 & 21,8 \\
\hline 12.02 .98 a 18.02 .98 & $15^{\mathrm{a}}$ & 92,7 & 30,8 & 21,8 \\
\hline
\end{tabular}

1 Total do período (Total of the period).

2 Média do período (Mean of the period).

ção recomendada $\left(91 \mathrm{~kg}\right.$ de $\mathrm{N}, 98 \mathrm{~kg}$ de $\mathrm{P}_{2} \mathrm{O}_{5}$ e $28 \mathrm{~kg}$ de $\mathrm{K}_{2} \mathrm{O} / \mathrm{ha}$, mais $84 \mathrm{~kg}$ de $\mathrm{S} / \mathrm{ha}$ ); e 4 - duas vezes a adubação recomendada (182 kg de N, $196 \mathrm{~kg}$ de $\mathrm{P}_{2} \mathrm{O}_{5}$ e $56 \mathrm{~kg}$ de $\mathrm{K}_{2} \mathrm{O} / \mathrm{ha}$, mais $168 \mathrm{~kg}$ de $\mathrm{S} / \mathrm{ha}$ ).

As parcelas experimentais constituíram-se de seis fileiras, com cinco metros de comprimento, espaçadas entre si de $0,8 \mathrm{~m}$, devidamente identificadas. Em cada parcela foram utilizadas para amostragem as duas fileiras centrais, descontadas de 0,5 metro em cada extremidade, resultando em uma área útil de $6,4 \mathrm{~m}^{2}$ (4,0 $\mathrm{m} \times 0,8 \mathrm{~m} \times 2)$.

O plantio foi realizado em 06.11.1997, e 10 dias após a germinação foi efetuado o desbaste nas parcelas, com a finalidade de se chegar à população de plantas por hectare recomendada para cada híbrido. Aos 20 dias após a germinação, foram realizadas uma capina manual e a primeira adubação de cobertura nos devidos tratamentos. Aos 35 dias após a germinação, efetuou-se a segunda adubação de cobertura.
Procedeu-se à colheita das plantas quando os grãos atingiram o estádio farináceo, ocasião em que as plantas da área útil das parcelas foram cortadas a uma altura de $15 \mathrm{~cm}$ em relação ao solo e pesadas em balança tipo "dinamômetro". Do total de plantas da parcela (área útil) foram retiradas 10 plantas inteiras, que foram picadas em picadeira 'Nogueira', modelo 'DPM 2', homogeneizadas, e retiradas amostras de aproximadamente $400 \mathrm{~g}$ do material original total para determinação do teor de matéria seca no momento da ensilagem e para avaliação químicobromatológica e nutricional dos híbridos. Foram também retiradas 20 plantas por parcela para análise da relação colmo/folha/panícula, por intermédio da pesagem, e colhidas amostras de cada uma dessas frações de aproximadamente $400 \mathrm{~g}$, para determinação da matéria seca (Gontijo Neto, 1999).

Utilizaram-se 80 silos experimentais, feitos de tubo de "PVC", com $10 \mathrm{~cm}$ de diâmetro e $50 \mathrm{~cm}$ de

Tabela 2 - Características químicas do solo da área experimental

Table 2 - Soil chemical characteristics of the experimental area

\begin{tabular}{|c|c|c|c|c|c|c|c|c|c|c|}
\hline $\begin{array}{c}\text { Profundidade } \\
\text { Depth }\end{array}$ & $\mathrm{pH}$ & $\mathrm{P}$ & $\mathrm{K}$ & $\mathrm{Ca}$ & $\mathrm{Mg}$ & SB & $\mathrm{H}+\mathrm{Al}$ & $\begin{array}{l}\text { CTC } \\
E C C\end{array}$ & $\mathrm{Al}$ & $\mathrm{V}$ \\
\hline $\mathrm{cm}$ & \multicolumn{3}{|c|}{$\mathrm{mg} / \mathrm{dm}^{3}$} & \multicolumn{5}{|c|}{$\mathrm{cmol} / \mathrm{dm}^{3}$} & \multicolumn{2}{|c|}{$\%$} \\
\hline $0-20$ & 5,6 & 9,8 & 83 & 3,1 & 1,2 & 4,51 & 5,0 & 9,51 & 0,1 & 47,4 \\
\hline $20-40$ & 5,6 & 6,0 & 29 & 3,0 & 1,0 & 4,00 & 5,2 & 9,26 & 0,0 & 43,8 \\
\hline
\end{tabular}


altura. Da área útil de cada parcela foi retirada uma amostra em quantidade suficiente para o enchimento dos silos, adotando-se uma densidade média de $887,17 \mathrm{~kg} / \mathrm{m}^{3}$, cuja compactação foi feita com um êmbolo de ferro e o fechamento com tampas de PVC lacradas com fita crepe e dotadas de válvula tipo "Bunsen". Os silos foram pesados por ocasião do enchimento e armazenados em local ventilado e fresco por um período aproximado de 60 dias.

Após a abertura de cada silo, seu conteúdo foi extraído e homogeneizado, sendo então retirada uma amostra, que foi pesada e acondicionada em saco de papel e colocada em estufa de ventilação forçada a $60-65^{\circ} \mathrm{C}$, por 72 horas. Em seguida, as amostras foram retiradas da estufa, deixadas à temperatura ambiente por uma hora e pesadas para determinação da matéria pré-seca. Posteriormente, procedeu-se à moagem das mesmas em moinho estacionário "Thomas Wiley", modelo 4, utilizando-se peneira com malha de $1 \mathrm{~mm}$, sendo guardadas em vidros com tampa para se realizar parte das determinações laboratoriais. Os teores de proteína bruta e dos constituintes da fração fibrosa e a digestibilidade in vitro da matéria seca foram determinados segundo metodologia descrita por Silva (1990). A metodologia para se determinar o teor de nitrogênio insolúvel em detergente ácido (NIDA) foi extraída de Licitra et al. (1996).

Foi utilizado um arranjo fatorial $5 \times 4$ (cinco híbridos x quatro doses de adubação) no delineamento experimental em blocos casualizados, com quatro repetições.

Os dados foram interpretados por meio de análise de variância e regressão, desdobrando-se a interação entre híbridos e doses de adubação independentemente da significância da mesma. As médias dos fatores qualitativos foram comparadas, utilizando-se o teste de Tukey, a 5\% de probabilidade. Os modelos foram escolhidos baseando-se na significância dos coeficientes de regressão, utilizando-se o teste "t", de Student, a $10 \%$ de probabilidade, e no coeficiente de determinação. Como ferramenta de auxílio às análises estatísticas foi utilizado o programa computacional SAEG - Sistema para Análises Estatísticas e Genéticas (UFV, 1995).

\section{Resultados e Discussão}

Proteina bruta e nitrogênio insolúvel em detergente ácido

$\mathrm{O}$ teor de $\mathrm{PB}$ diferiu $(\mathrm{P}<0,05)$ entre os híbridos, apresentando valores variando de 5,89 a $8,68 \%$, registrando-se maiores valores para a silagem do híbrido AG2005E em todas as doses de adubo (Tabela 3). Isso se explica pela maior participação de panícula nesse híbrido $(52,43 \%)$ e, portanto, maior teor de PB, conforme relatado por Gontijo Neto (1999). Maiores teores de PB em silagens com maior proporção de panículas foram também mencionados por Bruno et al. (1992), Genro et al. (1995), McCormick et al. (1995), Chaves et al. (1997) e Silva et al. (1999a). Os teores de PB observados no presente estudo (Tabela 3 ) encontram-se dentro da faixa de 4,5 a $8,2 \%$ registrada por White et al. (1988) para silagens de 80 híbridos de sorgo.

Apenas a silagem do híbrido AG2005E apresentou, em todas as doses de adubo, teor de PB superior a 7,0\%, nível mínimo considerado adequado para um funcionamento satisfatório da microbiota do rúmen.

$O$ teor de PB das silagens foi incrementado linearmente com o aumento nas doses de adubo aplicadas, excetuando-se a silagem do híbrido AG2002, cujo teor protéico médio foi de 6,26\%, conforme verifica-se na Figura 1. O efeito da adubação nitrogenada elevando o teor de PB da forragem é largamente documentado (Medeiros et al., 1979; Keady \& O'Kiely, 1996; Valk et al., 1996; McKenzie

Tabela 3 - Teores médios de proteína bruta $(\mathrm{PB})$ e nitrogênio insolúvel em detergente ácido (NIDA) das silagens nas doses de adubo testadas

Table 3 - Average contents of crude protein $(C P)$ and acid detergent insoluble nitrogen (ADIN) from silages under the tested fertilization levels

\begin{tabular}{|c|c|c|c|c|}
\hline \multirow[b]{2}{*}{$\begin{array}{l}\text { Híbridos } \\
\text { Hybrids }\end{array}$} & \multicolumn{4}{|c|}{$\begin{array}{l}\text { Doses de adubo (recomendação/ha) } \\
\text { Fertilization levels (recommendation/ha) }\end{array}$} \\
\hline & 0,0 & 0,5 & 1,0 & 2,0 \\
\hline & \multicolumn{4}{|c|}{$\mathrm{PB}(\% \mathrm{MS})$} \\
\hline AG2002 & $6,09^{b}$ & $6,37^{\mathrm{b}}$ & $6,30^{\mathrm{b}}$ & $6,27^{\mathrm{c}}$ \\
\hline AG2005E & $7,77^{\mathrm{a}}$ & $8,26^{\mathrm{a}}$ & $7,95^{\mathrm{a}}$ & $8,68^{\mathrm{a}}$ \\
\hline AGX202 & $6,65^{b}$ & $6,92^{\mathrm{b}}$ & $6,98^{b}$ & $7,57^{\mathrm{b}}$ \\
\hline AGX213 & $5,89^{\mathrm{b}}$ & $6,29^{b}$ & $6,47^{b}$ & $6,63^{\mathrm{c}}$ \\
\hline \multirow[t]{2}{*}{$\mathrm{AGX} 215$} & $6,55^{\mathrm{b}}$ & $6,81^{b}$ & $6,95^{\mathrm{b}}$ & $7,10^{b c}$ \\
\hline & \multicolumn{4}{|c|}{$\begin{array}{l}\text { NIDA }(\% \mathrm{~N} \text { total }) \\
\text { ADIN }(\% \text { total } N)\end{array}$} \\
\hline AG2002 & $9,95^{\mathrm{ab}}$ & $11,33^{\mathrm{a}}$ & $11,39^{a}$ & $11,28^{a}$ \\
\hline AG2005E & $6,43^{b}$ & $7,04 \mathrm{~b}$ & $6,38^{b}$ & $6,32^{\mathrm{b}}$ \\
\hline AGX202 & $10,42^{\mathrm{ab}}$ & $9,17^{\mathrm{ab}}$ & $10,58^{\mathrm{a}}$ & $10,60^{\mathrm{a}}$ \\
\hline AGX213 & $9,61^{\mathrm{ab}}$ & $12,27^{\mathrm{a}}$ & $12,66^{\mathrm{a}}$ & $12,08^{a}$ \\
\hline AGX215 & $12,17^{\mathrm{a}}$ & $9,89^{a b}$ & $10,42^{\mathrm{a}}$ & $11,92^{\mathrm{a}}$ \\
\hline
\end{tabular}

Médias na mesma coluna, dentro de cada variável, seguidas de letras minúsculas distintas, diferem $(P<0,05)$ pelo teste de Tukey.

Means in the same column, within each variable, followed by distinct small letters, differ $(P<.05)$ by Tukey test. 


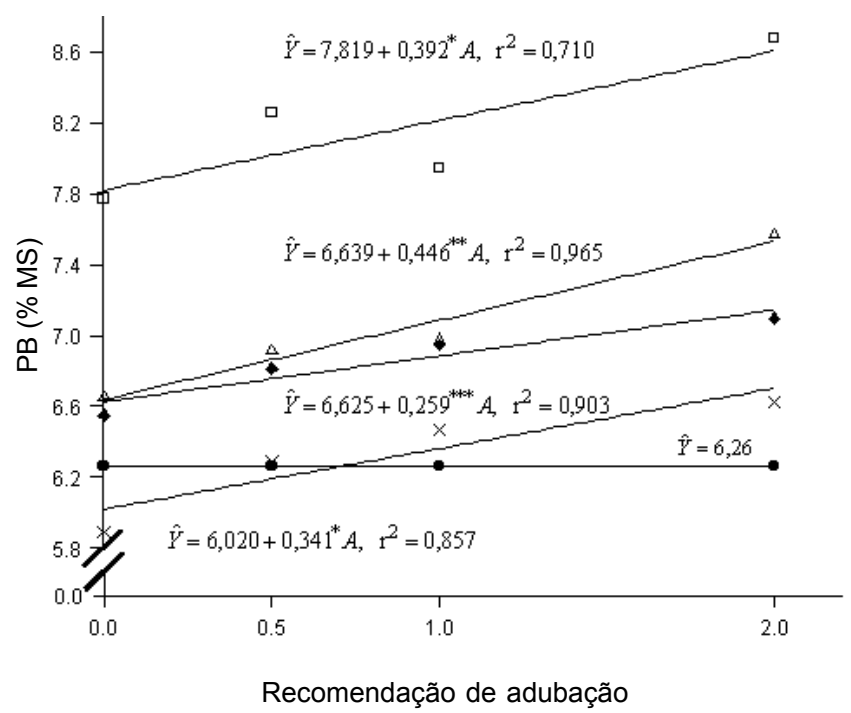

$-\mathrm{AG} 2002 \rightarrow \mathrm{AG} 2005 \mathrm{~A}-\mathrm{AGX} 202-\mathrm{AGX} 213-\mathrm{AGX} 215$

Figura 1 - Estimativa do teor de proteína bruta (PB) das silagens dos cinco híbridos, em função das doses de adubação (A).

Figure 1 - Crude protein $(C P)$ content estimation of the five hybrids silages as affected by the fertilization levels (A). ***,*** - $\mathrm{P}<0,1 ; 0,05$ e 0,01 , respectivamente, pelo teste $\mathrm{t}$.

$* * *,{ }^{* *},{ }^{*}-P<0.1, .05$ and .01 , respectively, by $t$ test.

et al., 1998). O híbrido que apresentou melhor resposta à adubação foi o AGX202, com aumento de 0,446 ponto percentual no teor de PB para cada elevação de uma unidade na recomendação de adubação $\left(91,98,28 \mathrm{~kg}\right.$ de $\mathrm{N}, \mathrm{P}_{2} \mathrm{O}_{5}$ e $\mathrm{K}_{2} \mathrm{O} /$ ha, respectivamente, mais $84 \mathrm{~kg}$ de $\mathrm{S} / \mathrm{ha}$ ).

Os teores de NIDA, por sua vez, variaram de 6,32 a $12,66 \%$ (Tabela 3). Apenas a silagem do híbrido AGX213 nas doses de 0,$5 ; 1,0$ e 2,0 vezes a adubação e do AGX215 no tratamento sem adubação apresentaram teores de NIDA ligeiramente superiores ao limite de $12 \%$ recomendado por Vilela (1998) para fermentação satisfatória. A silagem do AG2005E apresentou os menores teores de NIDA $(\mathrm{P}<0,05)$ em relação aos demais híbridos nas doses de 1,0 e 2,0 vezes a adubação recomendada (Tabela 3 ). Foi verificada correlação negativa $(r=-0,5711 * *)$ entre os teores de NIDA e de PB e correlação positiva com os carboidratos estruturais $(\mathrm{r}=0,3690 * *$ para FDN e $\mathrm{r}=0,5091^{*} *$ para FDA). Lavezzo et al. (1997) observaram teores de NIDA em silagens de milho inferiores a $2,5 \%$, enquanto o teor de fibra bruta das mesmas situou-se abaixo de $25 \%$. Por outro lado,
Gaggioti et al. (1992) relataram teores de NIDA superiores a $12 \%$, indo até $20,8 \%$ para silagens de sorgo cujos teores de FDA variaram de 35,0\% a $51,1 \%$, respectivamente.

Os teores de NIDA das silagens dos híbridos AG2005E, AGX202 e AG2002 não foram influenciados pelas doses de adubo, registrando-se, respectivamente, valores médios de 6,54; 10,19 e 10,99\%. Por sua vez, detectou-se efeito quadrático das doses de adubo para os teores de NIDA da silagem do híbrido AGX213 $\left(\hat{y}=9,758+5,257^{n s} A-2,060^{* * *} A^{2}, \mathrm{R}^{2}=0,953\right)$, com teor máximo $(13,12 \%)$ estimado para a dose correspondente a 1,28 vez a recomendação de adubação $(117,125$ e $36 \mathrm{~kg}$ de $\mathrm{N}, \mathrm{P}_{2} \mathrm{O}_{5}$ e $\mathrm{K}_{2} \mathrm{O} /$ ha, respectivamente, mais $108 \mathrm{~kg}$ de $\mathrm{S} / \mathrm{ha}$ ), e para a silagem do híbrido AGX215 $\left(\hat{y}=11,948-3,947^{n s} A+1,985^{* * *} A^{2}, \mathrm{R}^{2}=0,845\right)$, com teor mínimo $(9,99 \%)$ estimado para a dose correspondente a 0,99 vez a recomendação de adubação $(90,97$ e $28 \mathrm{~kg}$ de $\mathrm{N}, \mathrm{P}_{2} \mathrm{O}_{5}$ e $\mathrm{K}_{2} \mathrm{O} /$ ha, respectivamente, mais $83 \mathrm{~kg}$ de $\mathrm{S} / \mathrm{ha})$.

\section{Constituintes da parede celular}

Os teores de FDN das silagens diferiram $(\mathrm{P}<0,05)$ entre os híbridos, tendo variado de 47,40 a $64,34 \%$ (Tabela 4), situando-se dentro da faixa de 39,2 a $71,9 \%$ citada por White et al. (1988), avaliando a silagem de 80 cultivares de sorgo. A silagem do AG2005E apresentou o menor $(\mathrm{P}<0,05)$ teor de FDN no tratamento sem adubação. Não foi observado efeito significativo dos híbridos na dose de 2,0 vezes a recomendação de adubação (Tabela 4). As silagens dos híbridos forrageiros, de forma geral, não apresentaram grandes diferenças. Menor teor de FDN na silagem do sorgo de duplo-propósito (AG2005E) se explica pela maior participação de panícula nesse híbrido, conforme relatado por Gontijo Neto (1999), reduzindo a proporção de colmos e folhas, que apresentam mais elevado teor de fração fibrosa (Fisher \& Burns, 1987; Chaves et al., 1997).

Não se detectou efeito da adubação sobre os teores de FDN das silagens dos híbridos AG2002, AG2005E, AGX202 e AGX215, registrando-se, respectivamente, valores médios de 58,53; 58, 18; 55,60; e 48,93\%. Por sua vez, registrou-se efeito quadrático $(\mathrm{P}<0,1)$ da adubação para o teor de FDN da silagem do híbrido AGX213 (Figura 2), estimando-se valor mínimo de $53,98 \%$ para a dose de 1,45 vez a adubação recomendada $\left(132,142\right.$ e $41 \mathrm{~kg}$ de $\mathrm{N}, \mathrm{P}_{2} \mathrm{O}_{5}$ e $\mathrm{K}_{2} \mathrm{O} / \mathrm{ha}$, respectivamente, mais $122 \mathrm{~kg}$ de $\mathrm{S} / \mathrm{ha}$ ). Mullins et al. (1998) e Buxton et al. (1999) relataram redução no 
teor de FDN do sorgo com o aumento da fertilização nitrogenada e fosfatada, provavelmente pelo aumento no teor de carboidratos não-estruturais, diluindo a concentração dos componentes estruturais.

$\mathrm{Na}$ Tabela 4, encontram-se os teores médios de FDA das silagens que variaram de 27,23 a $37,66 \%$,

Tabela 4 - Teores médios de fibra em detergente neutro (FDN), fibra em detergente ácido (FDA), hemicelulose, celulose e lignina das silagens nas doses de adubo testadas

Table 4 - Neutral detergent fiber (NDF), acid detergent fiber $(A D F)$, hemicellulose, cellulose and lignin average contents of silages under the tested fertilization levels

\begin{tabular}{|c|c|c|c|c|}
\hline \multirow[b]{2}{*}{$\begin{array}{l}\text { Híbridos } \\
\text { Hybrids }\end{array}$} & \multicolumn{4}{|c|}{$\begin{array}{l}\text { Doses de adubo (recomendação/ha) } \\
\text { Fertilization levels (recommendation/ha) }\end{array}$} \\
\hline & 0,0 & 0,5 & 1,0 & 2,0 \\
\hline & \multicolumn{2}{|c|}{$N D F(\% D M)$} & & \\
\hline AG2002 & $56,96^{\mathrm{a}}$ & $53,97^{\mathrm{ab}}$ & $56,87^{\mathrm{ab}}$ & $54,61^{\mathrm{a}}$ \\
\hline AG2005E & $47,40^{\mathrm{b}}$ & $47,64^{\mathrm{b}}$ & $49,13^{b}$ & $51,57^{\mathrm{a}}$ \\
\hline AGX202 & $59,66^{\mathrm{a}}$ & $57,80^{\mathrm{a}}$ & $57,86^{\mathrm{ab}}$ & $57,42^{\mathrm{a}}$ \\
\hline AGX213 & $64,34^{\mathrm{a}}$ & $57,17^{\mathrm{a}}$ & $55,74^{\mathrm{ab}}$ & $55,29^{a}$ \\
\hline \multirow[t]{2}{*}{ AGX215 } & $59,09^{\mathrm{a}}$ & $55,46^{\mathrm{ab}}$ & $59,91^{\mathrm{a}}$ & $59,65^{\mathrm{a}}$ \\
\hline & \multicolumn{4}{|c|}{ FDA ( $\% \mathrm{MS})$} \\
\hline AG2002 & $32,31^{\mathrm{ab}}$ & $32,47^{\mathrm{ab}}$ & $31,42^{\mathrm{ab}}$ & $32,70^{\mathrm{ab}}$ \\
\hline AG2005E & $27,23^{\mathrm{b}}$ & $28,06^{\mathrm{b}}$ & $27,80^{\mathrm{b}}$ & $29,57^{b}$ \\
\hline AGX202 & $35,54^{\mathrm{a}}$ & $32,32^{\mathrm{ab}}$ & $34,38^{\mathrm{a}}$ & $33,92^{\mathrm{ab}}$ \\
\hline AGX213 & $35,29^{\mathrm{a}}$ & $34,63^{\mathrm{a}}$ & $34,23^{\mathrm{a}}$ & $34,85^{\mathrm{ab}}$ \\
\hline \multirow[t]{2}{*}{ AGX215 } & $37,20^{\mathrm{a}}$ & $33,57^{\mathrm{ab}}$ & $35,16^{\mathrm{a}}$ & $37,66^{\mathrm{a}}$ \\
\hline & \multicolumn{4}{|c|}{ Hemicelulose (\% MS) } \\
\hline AG2002 & $24,64^{\mathrm{a}}$ & $21,50^{\mathrm{a}}$ & $25,45^{\mathrm{a}}$ & $21,91^{\mathrm{a}}$ \\
\hline AG2005E & $20,17^{\mathrm{a}}$ & $19,59^{\mathrm{a}}$ & $21,33^{\mathrm{a}}$ & $21,99^{\mathrm{a}}$ \\
\hline AGX202 & $24,12^{\mathrm{a}}$ & $25,48^{a}$ & $23,47^{\mathrm{a}}$ & $23,50^{\mathrm{a}}$ \\
\hline AGX213 & $29,05^{\mathrm{a}}$ & $22,54^{\mathrm{a}}$ & $21,51^{\mathrm{a}}$ & $20,44^{\mathrm{a}}$ \\
\hline \multirow[t]{2}{*}{ AGX215 } & $21,89^{\mathrm{a}}$ & $21,89^{\mathrm{a}}$ & $24,75^{\mathrm{a}}$ & $21,99^{\mathrm{a}}$ \\
\hline & \multicolumn{4}{|c|}{ Celulose (\% MS) } \\
\hline AG2002 & $25,32^{\mathrm{ab}}$ & $24,98^{a}$ & $22,86^{\mathrm{a}}$ & $25,35^{\mathrm{a}}$ \\
\hline AG2005E & $21,50^{\mathrm{b}}$ & $21,98^{a}$ & $22,35^{\mathrm{a}}$ & $23,64^{\mathrm{a}}$ \\
\hline AGX202 & $26,64^{\mathrm{ab}}$ & $25,39^{a}$ & $25,42^{\mathrm{a}}$ & $26,06^{\mathrm{a}}$ \\
\hline AGX213 & $25,64^{\mathrm{ab}}$ & $26,55^{a}$ & $25,56^{\mathrm{a}}$ & $25,60^{\mathrm{a}}$ \\
\hline \multirow[t]{2}{*}{ AGX215 } & $27,72^{\mathrm{a}}$ & $25,82^{\mathrm{a}}$ & $26,41^{\mathrm{a}}$ & $29,06^{\mathrm{a}}$ \\
\hline & \multicolumn{4}{|c|}{$\begin{array}{l}\text { Lignina }(\% \mathrm{MS}) \\
\text { Lignin }(\% D M)\end{array}$} \\
\hline AG2002 & $6,33^{\mathrm{ab}}$ & $6,95^{\mathrm{a}}$ & $7,57^{\mathrm{a}}$ & $6,69^{a b}$ \\
\hline AG2005E & $4,81^{\mathrm{b}}$ & $5,15^{b}$ & $4,44^{b}$ & $5,02^{\mathrm{b}}$ \\
\hline AGX202 & $7,62^{\mathrm{a}}$ & $6,19^{a b}$ & $7,92^{\mathrm{a}}$ & $6,77^{\mathrm{a}}$ \\
\hline AGX213 & $7,99^{\mathrm{a}}$ & $6,64^{\mathrm{ab}}$ & $7,02^{\mathrm{a}}$ & $7,30^{\mathrm{a}}$ \\
\hline AGX215 & $7,64^{\mathrm{a}}$ & $6,68^{a b}$ & $7,42^{\mathrm{a}}$ & $7,50^{\mathrm{a}}$ \\
\hline
\end{tabular}

Médias na mesma coluna, dentro de cada variável, seguidas de letras minúsculas distintas, diferem $(P<0,05)$ pelo teste de Tukey.

Means in same column, within each variable, followed by distinct small letters, differ $(P<.05)$ by Tukey test. valores estes dentro da faixa de 27,1 a $49,4 \%$ observada por White et al. (1988). A silagem do híbrido AG2005E apresentou menores $(\mathrm{P}<0,05)$ teores de FDA, juntamente com a do AG2002, no tratamento sem adubação e na dose correspondente à adubação recomendada. Não se detectou efeito da adubação sobre os teores de FDA da maioria das silagens, registrando-se, respectivamente, valores médios de 34,$75 ; 34,04 ; 32,22$; e $28,17 \%$ para as silagens dos híbridos AGX213, AGX202, AG2002eAG2005E. Houve efeito quadrático $\left(\hat{y}=36,759-5,511^{n s} A+3,017^{* * *} A^{2}, \mathrm{R}^{2}=0,774\right)$ das doses de adubo sobre o teor de FDA da silagem do híbrido AGX215, com o teor mínimo (34,24\%) sendo estimado para a dose correspondente a 0,91 vez a recomendação de adubação $(83,89$ e $25 \mathrm{~kg}$ de $\mathrm{N}, \mathrm{P}_{2} \mathrm{O}_{5}$ e $\mathrm{K}_{2} \mathrm{O} / \mathrm{ha}$, respectivamente, mais $76 \mathrm{~kg}$ de $\mathrm{S} / \mathrm{ha}$ ). Mesmo nessa dose, os teores de FDA das silagens desse híbrido apresentaram-se bastante elevados, o que mostra seu baixo potencial nutritivo em qualquer dose de adubo.

Quanto à hemicelulose, que variou de 19,59 a $25,48 \%$ (Tabela 4), não se detectou diferença entre as silagens. Comparando-se os teores de hemicelulose dos híbridos por ocasião da ensilagem (Gontijo Neto, 1999) com aqueles de suas respectivas silagens, observou-se decréscimo, variando de 4,42 (AGX202) a 13,1 pontos percentuais (AG2005E). Isto indica que parte dessa fração foi hidrolisada pela ação de hemicelulases ou pela acidez do meio, contribuindo para a produção de ácidos orgânicos e o abaixamento do $\mathrm{pH}$, conforme verificado por Hunt et al. (1993), Rodriguez et al. (1997), Almeida Filho et al. (1999) e Silva et al. (1999b).

O teor de hemicelulose das silagens dos híbridos AGX202, AG2002, AGX215 e AG2005E não foi afetado pelas doses de adubo, registrando-se, respectivamente, valores médios de 24,14; 23,38; 22,63; e $20,77 \%$. No entanto, observou-se efeito quadrático $\left(\hat{y}=28,630-11,899^{* *} A+3,937^{*} A^{2}, \mathrm{R}^{2}=0,952\right)$ para o teor de hemicelulose da silagem do híbrido AGX213, estimando-se ponto de mínimo de $19,64 \%$ para a dose correspondente a $1,51 \mathrm{vez}$ a adubação recomendada $\left(137,148\right.$ e $42 \mathrm{~kg}$ de $\mathrm{N}, \mathrm{P}_{2} \mathrm{O}_{5}$ e $\mathrm{K}_{2} \mathrm{O} /$ ha, respectivamente, mais $127 \mathrm{~kg}$ de $\mathrm{S} / \mathrm{ha}$ ).

$\mathrm{O}$ teor de celulose das silagens, que variou de 21,50 a $29,06 \%$, diferiu $(\mathrm{P}<0,05)$ entre os híbridos apenas na ausência de adubação, conforme observa-se na Tabela 4. Esses valores estão próximos daqueles obtidos por Corrêa (1996) para silagens de híbridos de sorgo colhidos nos estádios de grãos pastoso e farináceo, que registraram variação de 23,32 a $28,19 \%$. As 
doses crescentes de adubo não afetaram os teores de celulose das silagens, registrando-se valores médios de 27,$25 ; 25,88 ; 25,84 ; 24,63 ;$ e $22,37 \%$ para as silagens dos híbridos AGX215, AGX202, AGX213, AG2002 e AG2005E, respectivamente.

$O$ teor de lignina das silagens também diferiu $(\mathrm{P}<0,05)$ entre os híbridos, tendo variado de 4,44 a $7,99 \%$ (Tabela 4). Nogueira (1995) e Corrêa (1996) registraram em silagens valores variando de 3,07 a $5,72 \%$ e de 2,5 a $5,1 \%$, respectivamente. A silagem do AG2005E apresentou menores teores $(\mathrm{P}<0,05)$ em relação ao AG2002 nas doses de 0,5 e 1,0 recomendação de adubação (Tabela 4). Isso é reflexo de sua maior proporção de panícula, acarretando menor proporção da fração fibrosa como um todo e de seus componentes. Quanto ao efeito das doses de adubo sobre o teor de lignina, observaram-se resultados bastante variáveis (Figura 2). Para as silagens dos híbridos AGX215, AGX202 e AG2005E não foi verificado efeito, com valores médios de 7,31; 7,12; e $4,85 \%$, respectivamente. Já para a silagem do híbrido AG2002 verificou-se efeito quadrático $(P<0,05)$, com ponto máximo de $7,46 \%$ de lignina na dose correspondente a 1,11 vez a adubação recomendada $(101,109$ e $31 \mathrm{~kg}$ de $\mathrm{N}, \mathrm{P}_{2} \mathrm{O}_{5}$ e $\mathrm{K}_{2} \mathrm{O} /$ ha, respectivamente, mais $93 \mathrm{~kg} \mathrm{de} \mathrm{S/ha).} \mathrm{Para} \mathrm{a} \mathrm{silagem} \mathrm{do} \mathrm{híbrido} \mathrm{AGX213} \mathrm{foi}$ observado efeito quadrático $(\mathrm{P}<0,1)$ com ponto mínimo igual a $6,70 \%$ na dose de 1,13 vezes a adubação recomendada $\left(103,111\right.$ e $32 \mathrm{~kg}$ de $\mathrm{N}, \mathrm{P}_{2} \mathrm{O}_{5}$ e $\mathrm{K}_{2} \mathrm{O} / \mathrm{ha}$, respectivamente, mais $95 \mathrm{~kg}$ de $\mathrm{S} / \mathrm{ha}$ ).

O efeito da adubação sobre o teor de carboidratos estruturais foi inconsistente, conforme observado também por Peyraud \& Astigarraga (1998). Se por um lado espera-se redução no teor dos constituintes fibrosos, devido à aceleração no metabolismo da planta com a elevação das doses de adubo aplicadas (Gontijo Neto, 1999), por outro a fração solúvel será a mais atingida com a fermentação no interior do silo, podendo anular o efeito benéfico da adubação sobre os compostos solúveis da forragem.

Digestibilidade "in vitro" da matéria seca e produção de matéria seca digestível

A DIVMS das silagens diferiu $(\mathrm{P}<0,05)$ entre os híbridos, tendo variado de 38,36 a 60,30\% (Tabela 5), observando-se valores mais elevados para a silagem do híbrido AG2005E em relação aos híbridos AGX202 e AGX215 no tratamento sem adubação, em relação ao AGX215 na dose correspondente a $0,5 \mathrm{vez}$ a adubação recomendada e ao AGX213 na dose correspondente a
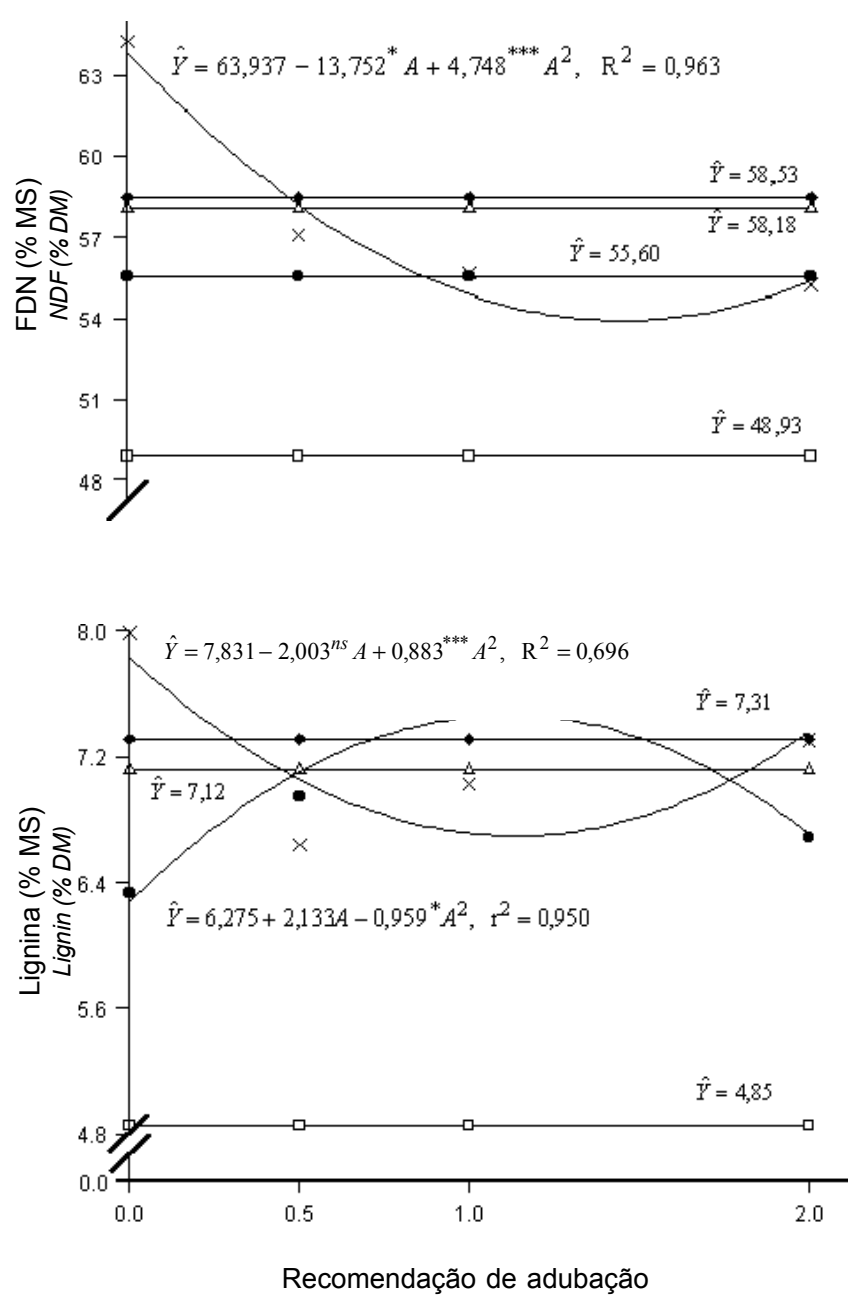

$\mathrm{AG} 2002 \rightarrow \mathrm{AG} 2005 \mathrm{~A}-\mathrm{AGX} 202 \rightarrow \mathrm{AGX} 213-\mathrm{AGX} 215$

Figura 2 - Estimativa dos teores de fibra em detergente neutro (FDN) e lignina das silagens dos cinco híbridos, em função das doses de adubação (A).

Figure 2 - Neutral detergent fiber (NDF) and lignin contents estimation of the five hybrids silages as affected by the fertilization levels $(A)$.

${ }^{* * *},{ }^{*}-\mathrm{P}<0,1$ e 0,05 , respectivamente, pelo teste $\mathrm{t}$. ${ }^{* * *},{ }^{*}-P<0.1$ and .05 , respectively, by $t$ test.

2,0 vezes a recomendação de adubação. Os valores observados estão dentro ou um pouco abaixo do intervalo de 44,6 a $62,1 \%$ citado por White et al. (1988) avaliando a silagem de 80 cultivares de sorgo.

A silagem do híbrido AG2005E apresentou, de forma geral, digestibilidade superior, possivelmente pela sua maior proporção de panícula $(52,43 \%)$ e menor proporção de colmos $(29,01 \%)$ e folhas (18,56\%), conforme relatado por Gontijo Neto (1999), 
refletindo no seu mais elevado teor protéico $(8,16 \%)$, menor teor de FDA $(28,17 \%)$ e menor teor de lignina (4,85\%). Efeitos similares da PB, FDA e lignina sobre a digestibilidade também foram observados por Schmid et al. (1976), Pedersen et al. (1982), Russell (1986), Hart (1987) e Pereira et al. (1993). De fato, constataram-se no presente estudo correlações significativas entre a DIVMS e os teores de PB $(\mathrm{r}=0,3641 * *)$, FDA $(\mathrm{r}=-0,4534 * *)$ e lignina $\left(\mathrm{r}=-0,5067^{* *}\right)$. Inversamente, a silagem do híbrido AGX215 apresentou a menor DIVMS (43,93\%), que pode ter sido influenciada pelo seu mais elevado teor de lignina $(7,31 \%)$ e FDA $(35,90 \%)$.

Na Figura 3, observa-se o comportamento da DIVMS das silagens em função da adubação. Verificase que a DIVMS das silagens dos híbridos AG2005E e AGX215 não foi influenciada pela adubação, registrando-se, respectivamente, valores médios de 58,92 e 43,93\%. Para o AGX202, observou-se resposta $(\mathrm{P}<0,05)$ linear à aplicação do adubo, estimando-se incremento de 4,36 pontos percentuais na DIVMS por unidade de aumento na dose de adubo recomendada $(91,98$ e $28 \mathrm{~kg}$ de $\mathrm{N}, \mathrm{P}_{2} \mathrm{O}_{5}$ e $\mathrm{K}_{2} \mathrm{O} /$ ha, respectivamente, mais $84 \mathrm{~kg}$ de $\mathrm{S} /$ ha). Buttrey et al. (1986) e Thompson \& Whitney (1998) também observaram aumento da DIVMS com a aplicação de doses crescentes de enxofre e nitrogênio, respectivamente. As silagens dos híbridos AGX213 e AG2002 apresentaram resposta quadrática $(\mathrm{P}<0,1)$ da DIVMS às doses crescentes de adubo. Efeito inconsistente da adubação sobre a digestibilidade também foi reportado por Valk et al. (1996), que justificaram haver outros fatores, como características agronômicas e idade à colheita, exercendo maior influência sobre essa variável.

A produção de matéria seca digestível (PMSD) das silagens diferiu $(\mathrm{P}<0,05)$ entre os híbridos, exceto na dose de 1,0 vez a adubação recomendada, tendo variado de 4,94 a 9,69 t/ha (Tabela 5). A silagem do AG2002 apresentou a maior $(\mathrm{P}<0,05)$ PMSD no tratamento sem adubação, juntamente com o AGX213. A silagem do AGX215 apresentou a menor $(\mathrm{P}<0,05)$ PMSD no tratamento sem adubação, juntamente com o AGX202, tendo sido grandemente afetada pela sua baixa DIVMS. A silagem do AG2005E, apesar de produzir menos (Gontijo Neto, 1999), obteve PMSD elevada, devido à sua elevada DIVMS.

Na Figura 3 encontra-se a estimativa da produção de matéria seca digestível (PMSD) das silagens em função das doses de adubo. Verifica-se que as silagens dos híbridos AG2005E e AGX202 não foram
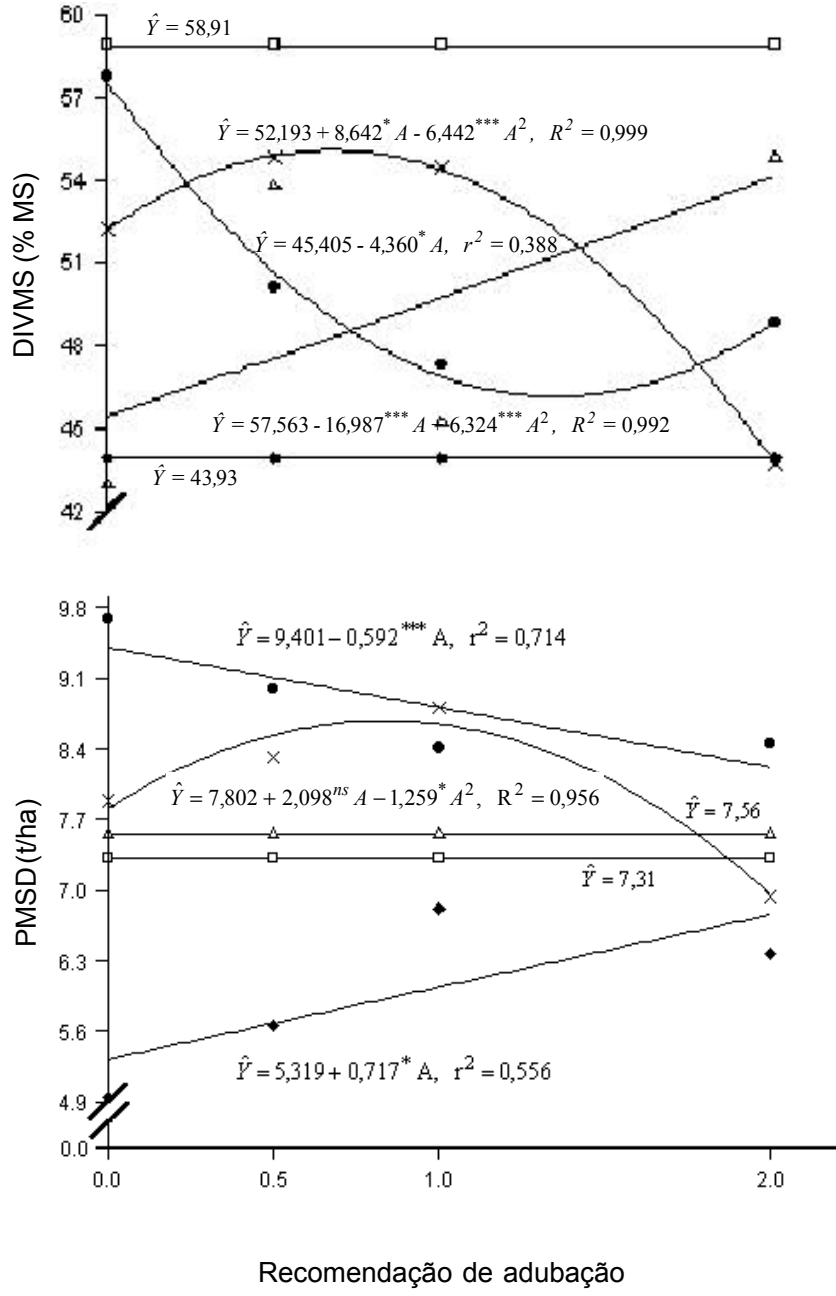

$-A G 2002 \rightarrow A G 2005 E \rightarrow A G X 202 \times A G X 213-A G X 215$

Figura 3 - Estimativa da digestibilidade in vitro da matéria seca e da produção de matéria seca digestível das silagens dos cinco híbridos, em função das doses de adubação (A).

Figure 3 - In vitro dry matter disappearance (IVDMD) and digestible dry matter production (DDMP) estimations of the five hybrids silages as affected by fertilization levels (A).

*** , ${ }^{*} \mathrm{P}<0,1$ e 0,05 , respectivamente, pelo teste $\mathrm{t}$. $* * * *{ }^{*}-P<0.1$ and .05 , respectively, by $t$ test.

influenciadas pelas doses crescentes de adubo, registrando-se, respectivamente, valores médios de 7,31 e 7,56 t/ha de MSD. Apenas a silagem do híbrido AGX215 apresentou efeito linear crescente $(\mathrm{P}<0,05)$, que está em consonância com os dados de Gontijo Neto (1999), o qual encontrou efeito linear crescente $(\mathrm{P}<0,01)$ sobre a PMSD média dos cinco híbridos estudados. 
Tabela 5 - Teores médios de digestibilidade in vitro da matéria seca (DIVMS) e produção de matéria seca digestível (PMSD) dos híbridos nas doses de adubo testadas

Table 5 - In vitro dry matter disappearance (IVDMD) and digestible dry matter production (DDMP) of silages under the tested fertilization levels

\begin{tabular}{|c|c|c|c|c|}
\hline \multirow[b]{2}{*}{$\begin{array}{l}\text { Híbridos } \\
\text { Hybrids }\end{array}$} & \multicolumn{4}{|c|}{$\begin{array}{l}\text { Doses de adubo (recomendação/ha) } \\
\text { Fertilization levels (recommendation/ha) }\end{array}$} \\
\hline & 0,0 & 0,5 & 1,0 & 2,0 \\
\hline & \multicolumn{4}{|c|}{ DIVMS (\%MS) } \\
\hline AG2002 & $57,77^{\mathrm{a}}$ & $50,10^{\mathrm{ab}}$ & $47,31^{\mathrm{a}}$ & $48,82^{\mathrm{ab}}$ \\
\hline AG2005E & $59,75^{\mathrm{a}}$ & $60,30^{\mathrm{a}}$ & $57,75^{\mathrm{a}}$ & $57,85^{\mathrm{a}}$ \\
\hline AGX202 & $43,01^{b c}$ & $53,81^{\mathrm{ab}}$ & $45,22^{\mathrm{a}}$ & $54,84^{\mathrm{ab}}$ \\
\hline AGX213 & $52,22^{\mathrm{ab}}$ & $54,83^{\mathrm{ab}}$ & $54,45^{\mathrm{a}}$ & $43,70^{\mathrm{b}}$ \\
\hline AGX215 & $38,36^{\mathrm{c}}$ & $42,16^{\mathrm{b}}$ & $48,71^{\mathrm{a}}$ & $46,47^{\mathrm{ab}}$ \\
\hline & \multicolumn{4}{|c|}{$\begin{array}{l}\operatorname{PMSD}(\mathrm{t} / \mathrm{ha}) \\
D D M P(t / h a)\end{array}$} \\
\hline AG2002 & $9,69^{\mathrm{a}}$ & $8,99^{\mathrm{a}}$ & $8,41^{\mathrm{a}}$ & $8,44^{\mathrm{ab}}$ \\
\hline AG2005E & $7,22^{b}$ & $7,56^{\mathrm{ab}}$ & $7,20^{\mathrm{a}}$ & $7,27^{\mathrm{ab}}$ \\
\hline AGX202 & $6,46^{\mathrm{bc}}$ & $8,09^{\mathrm{a}}$ & $6,93^{\mathrm{a}}$ & $8,78^{a}$ \\
\hline AGX213 & $7,88^{\mathrm{ab}}$ & $8,31^{\mathrm{a}}$ & $8,81^{\mathrm{a}}$ & $6,94^{\mathrm{ab}}$ \\
\hline AGX215 & $4,94^{\mathrm{c}}$ & $5,66^{\mathrm{b}}$ & $6,81^{\mathrm{a}}$ & $6,37^{b}$ \\
\hline
\end{tabular}

Médias na mesma coluna, dentro de cada variável, seguidas de letras minúsculas distintas, diferem $(\mathrm{P}<0,05)$ pelo teste de Tukey.

Means in same column, within each variable, followed by distinct small letters, differ $(P<.05)$ by Tukey test.

\section{Conclusões}

O valor nutritivo das silagens diferiu entre os híbridos, observando-se maior valor nutritivo para a silagem do AG2005E, enquanto as silagens do AG2002 e do AGX213 apresentaram maior produção de nutrientes por unidade de área.

O valor nutritivo das silagens foi afetado pelas doses de adubação, embora de forma inconsistente.

\section{Literatura Citada}

ALMEIDA FILHO, S.L.; FONSECA, D.M.; GARCIA, R. et al. Características agronômicas de cultivares de milho (Zea mays L.) e qualidade dos componentes e da silagem. Revista Brasileira de Zootecnia, v.28, n.1, p.7-13, 1999.

BRUNO, O.A.; ROMERO, L.A.; GAGGIOTI, L.A. et al. Cultivares de sorgos forrajeros para silaje. I. Rendimiento de materia seca y valor nutritivo de la planta. Revista Argentina de Producción Animal, v.12, n.2, p.157-162, 1992.

BUTTREY, S.A.; ALLEN, V.G.; FONTENOT, J.P. et al. Effect of sulfur fertilization on chemical composition, ensiling characteristics and utilization of corn silage by lambs. Journal of Animal Science, v.63, p.1236-1245, 1986.

BUXTON, D.R.; ANDERSON, I.C.; HALLAM, A. Performance of sweet and forage sorghum grown continuously, double- cropped with winter rye, or in rotation with soybean and maize. Agronomy Journal, v.91, p.93-101, 1999.

CHAVES, A.V.; OLIVEIRA, J.S.; ROCHA, V.S. et al. Comparação de cultivares de sorgo (Sorghum bicolor L. Moench) para produção de silagem. In: REUNIÃO DA SOCIEDADE BRASILEIRA DE ZOOTECNIA, 34., 1997, Juiz de Fora. Anais... Juiz de Fora: Sociedade Brasileira de Zootecnia, 1997.

COMISSÃO DE FERTILIDADE DO SOLO DO ESTADO DE MINAS GERAIS - CFSEMG. Recomendações para uso de corretivos e fertilizantes em Minas Gerais. $4^{\text {a }}$ Aproximação. Lavras: 1989. 159p.

CORRÊA, C.E.S. Qualidade das silagens de três híbridos de sorgo (Sorghum bicolor L.) em diferentes estádios de maturação. Belo Horizonte: Universidade Federal de Minas Gerais, 1996. 121p. Dissertação (Mestrado em Zootecnia) Universidade Federal de Minas Gerais, 1996.

FISHER, D.S.; BURNS, J.C. Quality analysis of summer-annual forages. I- Sample preparation methods and chemical characterization of forage types and cultivars. Agronomy Journal, v.79, p.236-242, 1987.

GAGGIOTI, M.C.; ROMERO, L.A.; BRUNO, O.A. Cultivares de sorgos forrajeros para silaje. II. Caracteristicas fermentativas y nutritivas de los silajes. Revista Argentina de Producción Animal, v.12, n.2, p.163-167, 1992.

GENRO, T.C.M.; QUADROS, F.L.F.; COELHO, L.G.M. et al. Produção e qualidade de silagens de híbridos de milho (Zea mays) e de híbridos de sorgo (Sorghum bicolor). Ciência Rural, v.25, n.3, p.461-464, 1995.

GONTIJO NETO, M.M. Rendimento e valor nutritivo de híbridos de sorgo (Sorghum bicolor (L.) Moench) sob níveis crescentes de adubação. Viçosa: Universidade Federal de Viçosa, 1999. 55p. Dissertação (Mestrado em Zootecnia) - Universidade Federal de Viçosa, 1999.

HART, S.P. Associative effects of sorghum silage and sorghum grain diets. Journal of Animal Science, v.64, p.1779-1789, 1987.

HUNT, C.W.; KEZAR, W.; HINMAN, D.D. et al. Effects of hybrid and ensiling with and without a microbial inoculant on the nutritional characteristics of whole-plant corn. Journal of Animal of Science, v.71, p.38-43, 1993.

KEADY, T.W.J.; O'KIELY, P. An evaluation of the effects or rate of nitrogen fertilization of grassland on silage fermentation, in-silo losses, effluent production and aerobic stability. Grass and Forage Science, v.51, p.350-362, 1996.

LAVEZZO, W.; CRESTE, C.R.; LAVEZZO, O.E.N.M. et al. Efeitos da retirada das espigas de milho sobre a produção e composição das plantas e silagens. In: REUNIÃO DA SOCIEDADE BRASILEIRA DE ZOOTECNIA, 34., 1997, Juiz de Fora. Anais... Juiz de Fora: Sociedade Brasileira de Zootecnia, 1997.

LICITRA, G.; HERNANDEZ, T.M.; Van SOEST, P.J. Standardization of procedures for nitrogen fractionation of ruminant feeds. Animal Feed Science and Technology, v.57, p.347-358, 1996.

McCORMICK, M.E.; MORRIS, D.R.; ACKERSON, B.A. et al. Ratoon cropping forage sorghum for silage: yield, fermentation, and nutrition. Agronomy Journal, v.87, p.952-957, 1995.

McKENZIE, R.H.; MIDDlETON, A.; SOlBERG, E. et al. Nitrogen and phosphorus optimize barley silage production. Better Crops, v.82, n.4, p.22-23, 1998.

MEDEIROS, R.B.; SAIBRO, J.C.; BARRETO, I.L. Efeito do nitrogênio e da população de plantas no rendimento e quali- 
dade do sorgo sodan (Sorghum bicolor (L.) Moench) $\mathbf{x}$ (Sorghum sudanense (Piper) Stapf.). Revista da Sociedade Brasileira de Zootecnia, v.8, n.1, p.75-87, 1979.

MULLINS, G.L.; ALLEY, S.E.; REEVES, D.W. Tropical maize response to nitrogen and starter fertilizer under strip and conventional tillage systems in southern Alabama. Soil and Tillage Research, v.45, p.1-15, 1998.

NOGUEIRA, F.A.S. Qualidade das silagens de híbridos de sorgo de porte baixo com e sem teores de taninos e de colmo seco e suculento, e seus padrões de fermentação, em condições de laboratório. Belo Horizonte: - Universidade Federal de Minas Gerais, 1995. 78p. Dissertação (Mestrado em Zootecnia) - Universidade Federal de Minas Gerais, 1995.

PEDERSEN, J.F.; GORZ, H.J.; HASKINS, F.A. et al. Variability for quality and agronomic traits in forage sorghum hybrids. Crop Science, v.22, p.853-856, 1982.

PEREIRA, O.G.; OBEID. J.A.; GOMIDE, J.A. et al. Produtividade de uma variedade de milho (Zea mays L.) e de três variedades de sorgo (Sorghum bicolor (L.) Moench) e o valor nutritivo de suas silagens. Revista da Sociedade Brasileira de Zootecnia, v.22, n.1, p.31-38, 1993.

PEYRAUD, J.L.; ASTIGARRAGA, L. Review of the effect of nitrogen fertilization on the chemical composition, intake, digestion and nutritive value of fresh herbage: consequences on animal nutrition and $\mathrm{N}$ balance. Animal Feed Science and Technology, v.72, p.235-259, 1998.

RESENDE, H. Cultura do milho e do sorgo para produção de silagem. Coronel Pacheco: Empresa Brasileira de Pesquisa Agropecuária, 1991. 107p. (EMBRAPA-CNPGL, Documentos, 51)

RODRIGUEZ, N.M.; BERNARDINO, M.L.A.; GONÇALVES, L.C. et al. Silagens de sorgo de porte médio com diferentes teores de tanino e suculência no colmo. II - Curva de fermentação de carboidratos e lignina. Arquivo Brasileiro de Medicina Veterinária e Zootecnia, v.49, n.3, p.317-329, 1997.

RUSSELL, J.R. Influence of harvest date on the nutritive value and ensiling characteristics of maize stover. Animal Feed Science and Technology, v.14, p.11-27, 1986.

SCHMID, A.R.; GOODRICH, R.D.; JORDAN, R.M. Relationship among agronomic characteristics of corn and sorghum cultivars and silage quality. Agronomy Journal, v.68, p.403-406, 1976.

SILVA, D.J. Análise de alimentos (métodos químicos e biológicos). 2.ed. Viçosa: Universidade Federal de Viçosa, 1990. $165 \mathrm{p}$.

SILVA, F.F.; GONÇALVES, L.C.; RODRIGUES, J.A.S. et al. Qualidade de silagens de híbridos de sorgo (Sorghum bicolor (L.) Moench) de portes baixo, médio e alto com diferentes proporções de colmo+folhas/panículas. 1. Avaliação do processo fermentativo. Revista Brasileira de Zootecnia, v.28, n.1, p.14-20, 1999a.
SILVA, F.F.; GONÇALVES, L.C.; RODRIGUES, J.A.S. et al. Qualidade de silagens de híbridos de sorgo (Sorghum bicolor (L.) Moench) de portes baixo, médio e alto com diferentes proporções de colmo+folhas/panículas. 2. Avaliação do valor nutritivo Revista Brasileira de Zootecnia, v.28, n.1, p.21-29, 1999b.

THOMPSON, C.A., WHITNEY, D.A. Long-term tillage and nitrogen fertilization in a West Central Great Plains wheatsorghum-fallow rotation. Journal of Production Agriculture, v.11, n.3, p.353-359, 1998.

TONANI, F.L. Valor nutritivo das silagens de sorgo (Sorghum bicolor (L.) Moench) em diferentes estádios de maturação dos grãos. Viçosa, MG: Universidade Federal de Viçosa, 1995. 56p. Dissertação (Mestrado em Zootecnia) - Universidade Federal de Viçosa, 1995.

UNIVERSIDADE FEDERAL DE VIÇOSA-UFV. SAEG - Sistema para análises estatística e genéticas. Versão 5.0. Viçosa, MG: 1995, 149p. (Manual do usuário).

VALK, H.; KAPPERS, I.E.; TAMMINGA, S. et al. In sacco degradation characteristics of organic matter, neutral detergent fibre and crude protein of fresh grass fertilized with different amounts of nitrogen. Animal Feed Science and Technology, v.63, p.63-87, 1996.

VILELA, D. Aditivos para silagem de plantas de clima tropical. In: SIMPÓSIO SOBRE ADITIVOS NA PRODUÇÃO DE RUMINANTES E NÃO-RUMINANTES/ REUNIÃO DA SOCIEDADE BRASILEIRA DE ZOOTECNIA, 35., 1998, Botucatu. Anais... Botucatu: Sociedade Brasileira de Zootecnia, 1998. p.73-108.

WHITE, J.; BOLSEN, K.; KIRCH, B. et al. Selecting forage sorghum cultivars for silage. Manhattan: Kansas Agricultural Experimental Station, 1988. 8p. (KSU. Report of Progress, 539).

ZAGO, C.P. Silagem de sorgo. In: SIMPÓSIO SOBRE NUTRIÇÃO DE BOVINOS: ALIMENTAÇÃO SUPLEMENTAR, 7., 1999, Piracicaba. Anais... Piracicaba: Fundação de Estudos Agrários "Luiz de Queiroz", 1999. p.47-68.
Recebido em: 19/09/00 Aceito em: 02/08/01 